\title{
Original Article \\ Investigation of Electroacupuncture and Manual Acupuncture on Carnitine and Glutathione in Muscle
}

\author{
Shizuo Toda \\ Department of Health Sciences, Kansai University of Health Sciences, 2-11-1 Wakaba, Kumatori, Sen-nan, Osaka-fu 590-0482, Japan \\ Correspondence should be addressed to Shizuo Toda, toda@kansai.ac.jp \\ Received 27 November 2008; Accepted 28 May 2009 \\ Copyright (C) 2011 Shizuo Toda. This is an open access article distributed under the Creative Commons Attribution License, which \\ permits unrestricted use, distribution, and reproduction in any medium, provided the original work is properly cited. \\ Electroacupuncture (EA) and manual acupuncture (MA) have therapeutic effects on muscle fatigue in muscle disease. The \\ deficiencies of carnitine and glutathione induce muscle fatigue. This report investigated the effects of EA and MA on carnitine \\ and glutathione in muscle. After the mice of EA group were fixed in the animal cage, right Zusanli (ST36) and Jiexi (ST41) were \\ acupunctured and stimulated with uniform reinforcing and reducing method by twirling the acupuncture needle for 15 min. And \\ then, the needle handles were connected to an electric stimulator for stimulating the acupoint with dense-sparse waves. After the \\ mice of MA group were fixed in an animal cage, right ST36 and ST41 were acupunctured and allowed for 15 min. The mice of \\ normal control group were not acupunctured and stimulated for $15 \mathrm{~min}$. The mice of all groups were killed for collecting muscle \\ tissue $1 \mathrm{~h}$ after the final treatment. Carnitine and glutathione in homogenate of muscle tissue were determined with carnitine \\ (Kainos Laboratories Co., Tokyo, Japan) and glutathione assay kit (Dojin Chemicals Co., Kumamoto, Japan). Carnitine level in \\ muscle tissue of MA group was significantly higher than those of EA group and normal control group. Carnitine level in muscle \\ tissue of EA group was not significantly different from that of normal control group. Glutathione levels in muscle tissue of EA \\ group and MA group were significantly higher than that of normal control group. This report presented that carnitine in muscle \\ is increased by MA, and not increased by EA, and that glutathione in muscle is increased by EA and MA.
}

\section{Introduction}

Muscle disease are associated with contractures, craps muscle stiffness and deep muscle aching. These phenomena relate to muscle fatigue [1].

The pathogenesis of fatigue in muscle condition has the relation carnitine and glutathione. Carnitine appears to improve force of muscle while stimulated in situ. This effect of carnitine is acute and stereospecific [2]. In energy metabolism, carnitine has a major role in the translocation of long-chain fatty acids into the mitochondrial matrix for subsequent $\beta$-oxidation, and in the regulation of the mitochondrial in skeletal muscle [3]. The alternative explanation on skeletal muscle fatigue has been considered the effects of reactive oxygen species (ROS). ROS scavenger as glutathione reduces skeletal muscle fatigue [4].

Acupunctures as electroacupuncture (EA) and manual acupuncture (MA) can protect cells from injury of acute spots and maintain the functions of mitochondria so as to delay fatigue, prolong working time of muscles [5]. It has been unclear the difference of effect between EA and MA on muscle.
This report investigates the effects of EA and MA on carnitine and glutathione in muscle.

\section{Materials and Methods}

2.1. Grouping of Animals. Male ddY mice weighing between 25 and $30 \mathrm{~g}, 6-$ to 7 -weeks-old were obtained from SLC, Shizuoka, Japan. They were housed in a room maintained at $25^{\circ} \mathrm{C}$ with a relative humidity of $60 \%$ and given standard laboratory chow and water ad libitum in the Laboratory Animal Center of Kansai University of Health Sciences. The care and use of animals followed the guidelines of Laboratory Animals in Kansai University of Health Sciences. Fifteen rats were allocated to three groups. They were EA, MA and normal control groups. There were five mice in one group.

2.2. Acupoint and Method of Acupuncture. The animal cage (Orientalgiken Co., Tokyo, Japan) has five holes for four limbs and a tail. During acupuncture and stimulation, mice were maintained in the animal cage with right hind limbs taken out and fastened to the wall of the animal cage with 
tape. In EA group and MA group, the mice were acupunctured by inserting $3 \mathrm{~mm}$ deep at right Zsusanli (ST36) and Jiexi (ST41) with sterilized disposable stainless steel acupuncture needles $(0.25 \times 30 \mathrm{~mm}$, Seirin Co., Shizuoka, Japan). Mice ST36 were determined according to human ST36 which is located on the tibialis anterior muscle [6]. Mice ST41 were determined according to human ST41 which is located between two tendons on the dorsum of the foot which are more distinct when the ankle is dorsiflexion, and is the midpoint of the line connecting the prominences of the lateral malleolus and medical malleolus [6].

After the mice of EA group were fixed in the animal cage, right ST36 and ST41 were acupunctured and stimulated with uniform reinforcing and reducing method by twirling the acupuncture needle for $15 \mathrm{~min}$. And then the needle handles were connected to Neurostimulator (Neuro Medical Co. Osaka, Japan) for stimulating the acupoints with densesparse waves, frequency of $3 \mathrm{~Hz}$ for $30 \mathrm{~min}$, and amplitude: positive pulse $50 \mathrm{~V}$; negative pulse $25 \mathrm{~V}$.

After the mice of MA group were fixed in the animal cage, right ST36 and ST41 were acupunctured and allowed for $15 \mathrm{~min}$.

The mice of normal control group were not both acupunctured and stimulated.

\subsection{Collection of Muscle Samples and Measurements of Carni-} tine and Glutathione. The mice of EA group and MA group were killed for the treated skeletal muscles between lateral ST36 and ST41 $1 \mathrm{~h}$ after the treatment. The treated skeletal muscles were removed from the mice immediately after killing and were subjected to homogenization in ice-cold $50 \mathrm{mM}$ Tris- $\mathrm{HCl}$ buffer at pH 7.4 using a Potter-Elvehjem homogenizer. Carnitine and glutathione in homogenate of muscle tissue were determined with carnitine (Kainos Laboratories Co., Tokyo, Japan) and glutathione assay kit (Dojin Chemicals Co., Kumamoto, Japan).

2.4. Statistical Analysis. Data were expressed as mean \pm standard error and analyzed by Mann-Whitney $U$-test.

\section{Results}

Carnitine level in muscle tissue of MA group was significantly higher than those of EA group and normal control group $(P<.001)$. Carnitine level in muscle tissue of EA group was not significantly different from that of normal control group. Glutathione levels in muscle tissues of EA group and MA group were significantly higher than that of normal control group $(P<.001)$ (Table 1$)$.

\section{Discussion}

Acupuncture at ST36 and ST41 has been shown to be a better therapeutic effect for muscle fatigue, problems of constriction and myofacial pain of leg muscle [7]. Acupuncture at these acupoints reduces the tibialis anterior electromyographymuscle activity [8]. Acupuncture leads to analgesia and improvement in other somatic sympotoms
TABle 1: Comparison of carnitine and glutathione contents in muscle tissues between three groups (mean \pm standard error, $\mu \mathrm{mol} /$ g).

\begin{tabular}{lll}
\hline Groups & Carnitine & Glutathione \\
\hline Electrocupuncture group & $363.33 \pm 46.33$ & $34.11 \pm 2.03^{\mathrm{a}}$ \\
Manual acupuncture group & $654.17 \pm 50.52^{\mathrm{b}, \mathrm{a}}$ & $31.38 \pm 1.34^{\mathrm{a}}$ \\
Normal control group & $346.67 \pm 53.41$ & $26.79 \pm 3.18$ \\
\hline
\end{tabular}

${ }^{\mathrm{a}} P<.001$ versus normal control group; ${ }^{\mathrm{b}} \mathrm{P}<.001$ versus electroacupuncture group.

of fibromyalgia on a single-site, single-blind, randomized trial [9]. Acupuncture has been useful therapy on muscle symptoms.

Carnitine therapy is effective in ameliorating fatigue in celiac disease on randomized double-blind versus placebo parallel study [10]. Carnitine can directly improve the fatigue characteristics of muscles enriched in type I fiber [11]. More than 95\% of carnitine in the body exists within skeletal muscle tissue [12]. Carnitine transfer fatty acids to the mitochondria and involves energy metabolism. Carnitine deficiencies induce muscle fatigue and myopathy [13]. Carnitine in blood and brain showed to be increased by acupuncture [14]. Contraction-induced production of ROS causes oxidative stress to skeletal muscle [15]. ROS contribute to muscle fatigue. Anti-oxidant as glutathione has been shown to lessen oxidation of cellular constituents and delay muscle fatigue [16]. The addition of anti-oxidant as GSH can improve muscle performance [17]. EA has the protective effects on mitochondria of cortical neuron in acute cerebral ischemia-reperfusion rats. It has been speculated that these results are related with anti-oxidant as glutathione [18]. These following findings showed that the effects of acupuncture may be related with carnitine and glutathione.

EA and MA can increase $\mathrm{Ca}^{2+}$ content and $\mathrm{Ca}^{2+}$-ATPase activity in sarcoplasmic reticulum of skeletal muscle cells in motor fatigue rats [19]. EA is superior to MA in training patients with tennis elbow [20]. These findings showed that these differences of effects between EA and MA may depend on the condition of muscle.

The presented results suggested that increases of carnitine by MA and of glutathione by EA and MA may improve muscle disease. The differences of carnitine level between EA and MA may depend on acupuncture stimulation. This investigation should be progressed further.

\section{Conclusion}

EA and MA have been shown to have therapeutic effects on muscle fatigue in muscle disease. This report presented that carnitine in muscle is increased by acupuncture, and not increased by EA, and that glutathione in muscle is increased by EA and MA.

\section{References}

[1] H. Anthony, V. Schapira, and C. Robert, Muscle Pain and Fatigue. Muscle in Diseases, Butterworth Heinemann, Boston, Mass, USA, 1999. 
[2] M.-L. Dubelaar, C. M. H. B. Lucas, and W. C. Hulsmann, "Acute effect of L-carnitine on skeletal muscle force tests in dogs," American Journal of Physiology, vol. 260, no. 2, pp. E189-E193, 1991.

[3] F. B. Stephens, D. Constantin-Teodosiu, and P. L. Greenhaff, "New insights concerning the role of carnitne in the regulation o fuel metabolism in skeletal muscle," The Journal of Physiology, vol. 581, pp. 431-444, 2007.

[4] D. G. Allen, G. D. Lamb, and H. Westerblad, "Skeletal muscle fatigue: cellular mechanisms," Physiological Reviews, vol. 88, no. 1, pp. 287-332, 2008.

[5] M. Gao, H. Y. Yang, T. Y. Liu, and I. Kuai, "Effects of manual acupuncture and electroacupuncture on mitochondria of skeletal muscle cells in rats of acute swimming exercise," Zhongguo Zhen Jiu, vol. 25, pp. 421-424, 2005.

[6] World Health Organization, WHO Standard Acupuncture Point Locations in the Western Pacific Region, World Health Organization, 2008.

[7] H. Gellman, "Acupuncture physical medicine," in Acupuncture Treatment for Musculoskeletal Pain, pp. 162-180, Taylor \& Francis, Boston, Mass, USA, 2002.

[8] L. A. Costa and J. E. de Araujo, "The immediate effects of local and adjacent acupuncture on the tibialis anterior muscle: a human study," Chinese Medicine, vol. 3, article no. 17, pp. 1-6, 2008.

[9] R. E. Harris, X. Tian, D. A. Williams et al., "Treatment of fibromyalgia with formula acupuncture: investigation of needle placement, needle stimulation, and treatment frequency," Journal of Alternative and Complementary Medicine, vol. 11, no. 4, pp. 663-671, 2005.

[10] C. Ciacci, G. Peluso, E. Iannoni et al., "L-Carnitine in the treatment of fatigue in adult celiac disease patients. A pilot study," Digestive and Liver Disease, vol. 39, no. 10, pp. 922-928, 2007.

[11] E. P. Brass, A. M. Scarrow, L. J. Ruff, K. A. Masterson, and E. Van Lunteren, "Carnitine delays rat skeletal muscle fatigue in vitro," Journal of Applied Physiology, vol. 75, no. 4, pp. 15951600, 1993.

[12] E. P. Brass, "Pharmacokinetic considerations for the therapeutic use of carnitine in hemodialysis patients," Clinical Therapeutics, vol. 17, no. 2, pp. 176-185, 1995.

[13] C. Jacob and F. Belleville, "L-carnitine: metabolism, functions and value in pathology," Pathologie Biologie, vol. 40, no. 9, pp. 910-919, 1992.

[14] S. Toda, "Effects of electroacupuncture on carnitine in serum and brain," World Journal of Acupuncture-Moxibustion, vol. 17, pp. 22-24, 2007.

[15] L. L. Ji, "Modulation of skeletal muscle antioxidant defense by exercise: role of redox signaling," Free Radical Biology and Medicine, vol. 44, no. 2, pp. 142-152, 2008.

[16] L. F. Ferreira and M. B. Reid, "Muscle-derived ROS and thiol regulation in muscle fatigue," Journal of Applied Physiology, vol. 104, no. 3, pp. 853-860, 2008.

[17] S. K. Powers and K. Hamilton, "Antioxidants and exercise," Clinics in Sports Medicine, vol. 18, no. 3, pp. 525-536, 1999.

[18] Z. B. Liu and W. M. Niu, "Protective effect of electroacupuncture of "xiusannzhen" on neuronal mitochondria in rats with acute cerebral ischemia-reperfusion injury," Zhen Ci Yan Jiu, vol. 32, pp. 163-166, 2007.

[19] M. Gao, H. Y. Yang, K. Le, T. Y. Liu, and X. J. Gu, "Effects of manual acupuncture and electroacupuncture on $\mathrm{Ca}^{2+}$ content and $\mathrm{Ca}^{2+}$-ATPase activity in sarcoplasmic reticulum of skeletal muscle cells in rats during acute swimming exercise," Zhen Ci Yan Jiu, vol. 33, pp. 13-16, 2008.
[20] P. Tsui and M. C. Leung, "Comparison of the effectiveness between manual acupuncture and electro-acupuncture on patients with tennis elbow," Acupuncture and ElectroTherapeutic Research, vol. 27, pp. 107-117, 2002. 


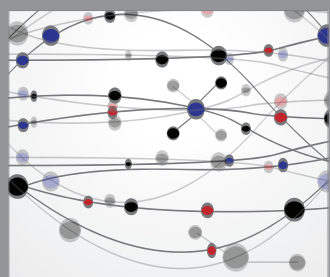

The Scientific World Journal
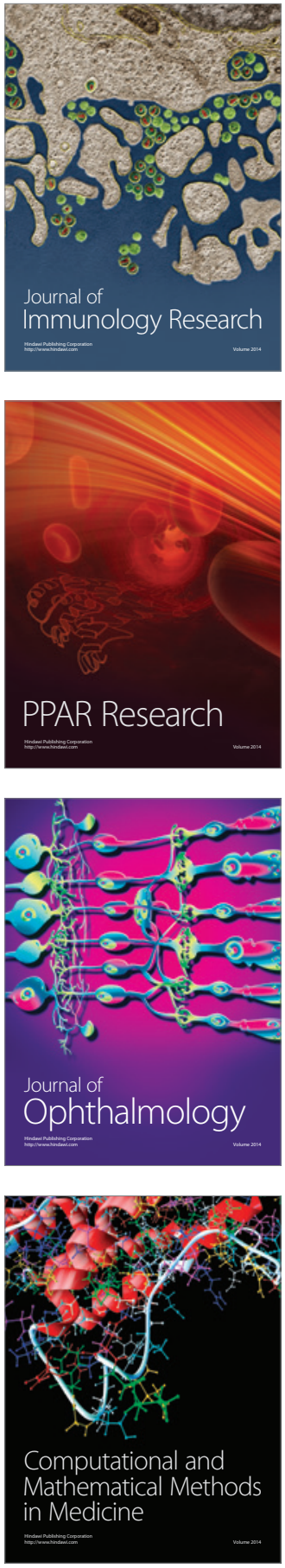

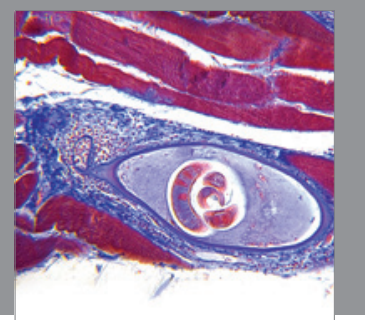

Gastroenterology

Research and Practice
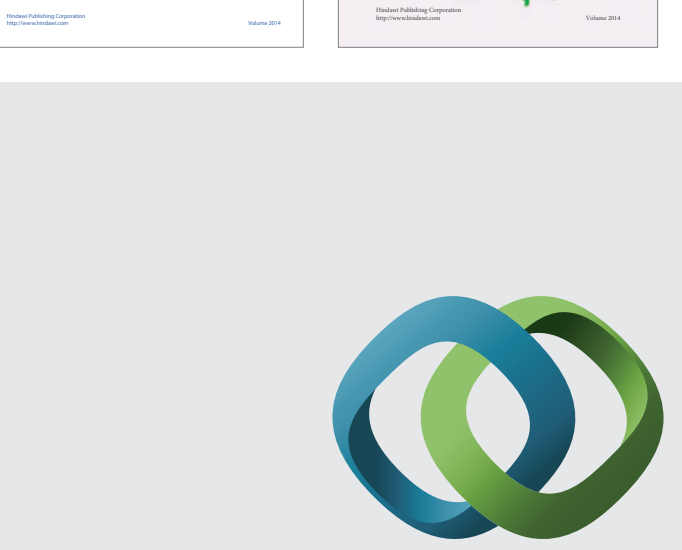

\section{Hindawi}

Submit your manuscripts at

http://www.hindawi.com
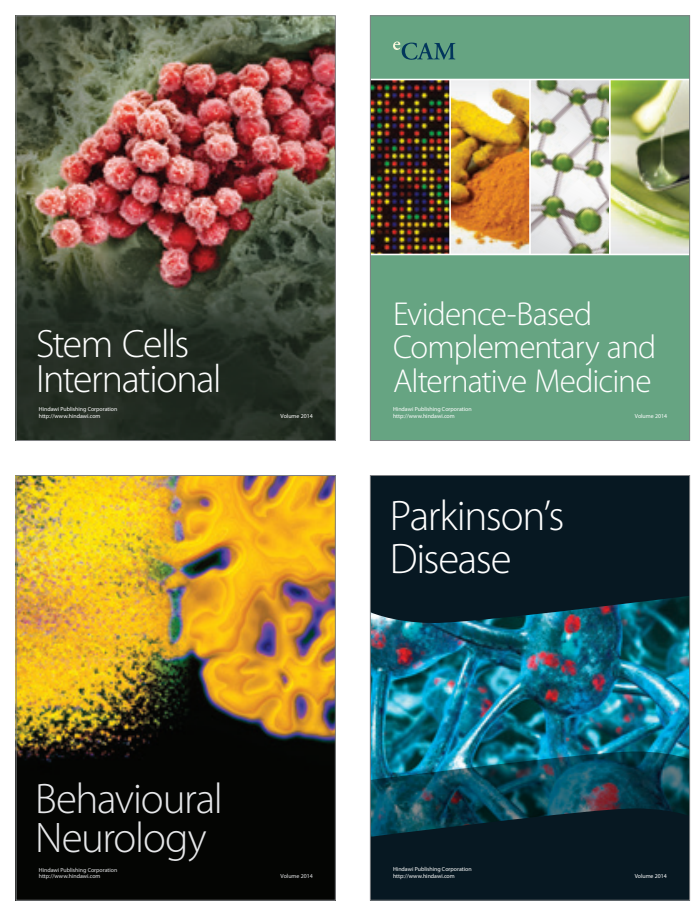

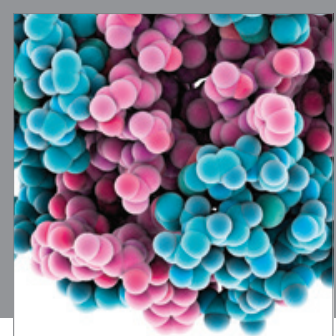

Journal of
Diabetes Research

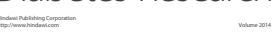

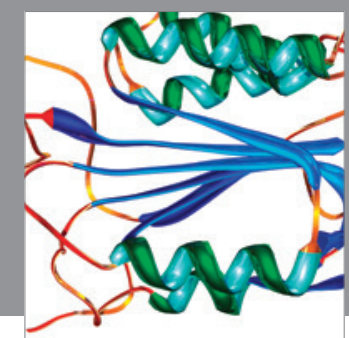

Disease Markers
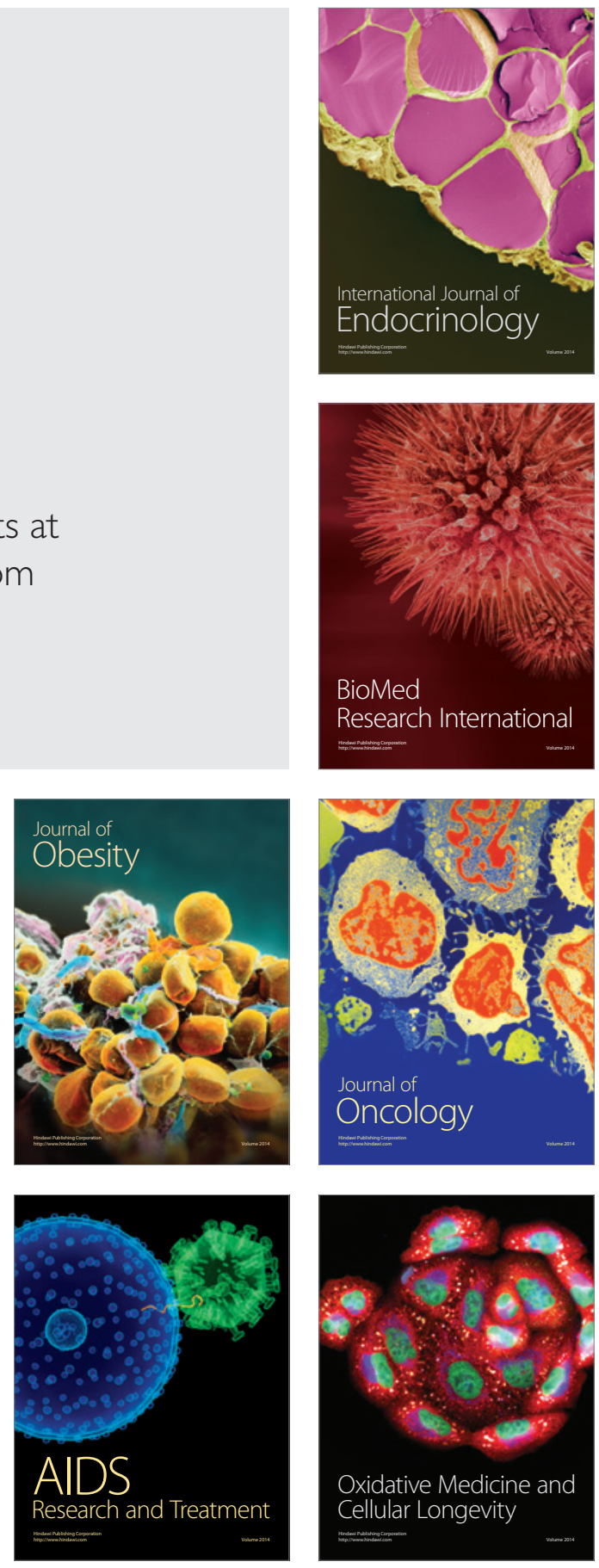